Check for updates

Cite this: RSC Adv., 2019, 9, 7833

\title{
Thermal conductivity of polyvinylpolymethylsiloxane aerogels with high specific surface area
}

\author{
Lukai Wang, Junzong Feng, Yonggang Jiang, Liangjun Li and Jian Feng (D) *
}

\begin{abstract}
The traditional $\mathrm{SiO}_{2}$ aerogels are difficult to apply in the fields of energy storage and heat insulation due to their poor mechanical properties. In order to deal with this issue, the polyvinylpolymethylsiloxane aerogel (PVPMSA) materials with fine mechanical flexibility and excellent thermal insulation properties are suitable substitutions. In this paper, the double cross-linking organic-inorganic hybrid PVPMSAs were prepared through the processes of free radical polymerization and hydrolytic polycondensation. The internal silica network reinforced with aliphatic hydrocarbons has significantly improved the mechanical properties and acquired a high specific surface area, reaching up to $1218 \mathrm{~m}^{2} \mathrm{~g}^{-1}$. Furthermore, the thermal conductivity of monolithic PVPMSAs has been investigated by changing the density and environmental conditions. Results show that PVPMSAs at $25^{\circ} \mathrm{C}$ in $5 \mathrm{~Pa}$ have a thermal conductivity as low as $14.69 \mathrm{~mW} \mathrm{~m}^{-1} \mathrm{~K}^{-1}$, and the solid thermal conductivity shows a flat growth with the increase of density. Meanwhile, the nanosize pores could significantly inhibit the heat transfer of gas. As for the radiative thermal conductivity, it is greatly affected by temperature. All these results obtained from this paper would help us to design thermal insulators reasonably.
\end{abstract}

Received 22nd December 2018

Accepted 4th March 2019

DOI: $10.1039 / c 8 \mathrm{ra10493j}$

rsc.li/rsc-advances containing organic functional groups as precursors, such as organoalkoxysilanes $\mathrm{R}_{n} \mathrm{Si}\left(\mathrm{OR}^{\prime}\right)_{4-n}$ and organo-bridged alkoxysilanes $\left(\mathrm{R}^{\prime} \mathrm{O}\right)_{3} \mathrm{Si}-\mathrm{R}-\mathrm{Si}\left(\mathrm{OR}^{\prime}\right)_{3}$ and $\left(\mathrm{R}^{\prime} \mathrm{O}\right)_{2}\left(\mathrm{CH}_{3}\right) \mathrm{Si}-\mathrm{R}-\mathrm{Si}\left(\mathrm{CH}_{3}\right)\left(\mathrm{OR}^{\prime}\right)_{2}$, where $\mathrm{R}$ and $\mathrm{R}^{\prime}$ are organic functional groups. ${ }^{16,20,21}$ Thus, silica aerogels reinforced with organic components possess better mechanical property, especially for the double cross-linking polyorganosiloxane aerogels. These aerogels prepared with polyorganoalkoxysilane precursors obtained by radical polymerization of vinyl monomers exhibit excellent bending and compression flexibility. Recently, Zu's group $^{22-24}$ has first prepared the doubly cross-linked polyvinylpolymethylsiloxane aerogels (PVPMSAs), which has solved the brittleness of traditional silica aerogels. This strategy can significantly enhance the skeleton structure of aerogels and prevent aerogels from cracking in the drying process. What's more important is that the introduction of organic components would not significantly increase the thermal conductivity of organic-inorganic hybrid aerogels.

At present, the thermal conductivity of PVPMSAs has not been systematically investigated, especially in different circumstances. And for designing the high-performance insulators, we have taken place a detailed study on PVPMSAs and reported the test results in this paper. Firstly, we have synthesized polyvinylmethyldimethoxysilane (PVMDMS) precursor using vinylmethyldimethoxysilane (VMDMS) as a monomer by Zu's method. ${ }^{22}$ Then, using strong base tetramethylammonium hydroxide (TMAOH) as a catalyst, PVPMSAs were obtained by the sol-gel process and $\mathrm{CO}_{2}$ supercritical drying. Finally, this work exhibits the surface morphology, SSA, pore size
Science and Technology on Advanced Ceramic Fibers and Composites Laboratory, College of Aerospace Science and Engineering, National University of Defense Technology, 109 De Ya Rd, Changsha, Hunan, 410073, People's Republic of China. E-mail:fengj@nudt.edu.cn 
distribution, and thermal conductivity of PVPMSAs. At the same time, the effects of different temperatures $\left(25-90{ }^{\circ} \mathrm{C}\right)$, gas pressure (5 $\mathrm{Pa}-100 \mathrm{kPa}$ ), and atmospheres (air, argon, and $\mathrm{CO}_{2}$ ) on the thermal conductivity were also systematically studied in this paper.

\section{Experimental section}

\subsection{Materials}

Vinylmethyldimethoxysilane (purity: 97\%), di-tert-butyl peroxide (purity: 97\%), tetramethylammonium hydroxide pentahydrate (purity: 97\%), benzyl alcohol (GC), ethanol (AR), and distilled water were purchased from Shanghai Aladdin Biochemical Technology Co., Ltd. All reagents in this work were used as received without further purification.

\subsection{Preparation of PVPMSAs}

Mixture of VMDMS and di-tert-butyl peroxide (DTBP) with a molar ratio of $10: 1$ was added to the hydrothermal reactor, and argon was injected into the hydrothermal reactor three times to remove air. Then, the hydrothermal reactor was sealed and heated at $120{ }^{\circ} \mathrm{C}$ for $72 \mathrm{~h}$. After the reaction, the transparent PVMDMS can be obtained by cooling to room temperature, the weight-average molecular weight of which can reach up to 13982 , and the degree of polymerization is up to 105 . For the sol-gel process, the molar ratio of precursor PVMDMS to benzyl alcohol $(\mathrm{BzOH})$ was controlled at $\mathrm{BzOH} / \mathrm{Si}=3.0-7.0 \mathrm{~mol} \mathrm{~mol}^{-1}$, and the uniformly dispersed transparent solution was obtained by slow stirring. Then, tetramethylammonium hydroxide $(\mathrm{TMAOH}) / \mathrm{Si}=$ $0.05 \mathrm{~mol} \mathrm{~mol}^{-1}$ and deionized water $\left(\mathrm{H}_{2} \mathrm{O}\right) / \mathrm{Si}=2.0 \mathrm{~mol} \mathrm{~mol}^{-1}$ were added, and the homogeneous sol was obtained by slowly stirring for $10 \mathrm{~min}$. After that, the sol was transported into a plastic container with a lid and sealed. And the gel was obtained at $50{ }^{\circ} \mathrm{C}$ for $30 \mathrm{~min}$. Then the gel was aged at $80^{\circ} \mathrm{C}$ for $4 \mathrm{~d}$ in an oven, and after 4 times of ethanol solvent exchange (each $24 \mathrm{~h}$ ) at $60{ }^{\circ} \mathrm{C}$ to wash and remove the residuals, the orange gel turns to a transparent gel. Finally, PVPMSAs were obtained after experiencing $\mathrm{CO}_{2}$ supercritical drying $\left(55^{\circ} \mathrm{C}, 15 \mathrm{MPa}\right)$.

\subsection{Characterizations}

The bulk density $(\rho)$ was determined by measuring the weight and volume of samples. The weight-average molecular weight $\left(M_{\mathrm{w}}\right)$ and polydispersity $\left(M_{\mathrm{w}} / M_{\mathrm{n}}\right)$ of PVMDMS were determined by gel permeation chromatography (GPC) system (PL-GPC50, Agilent, Germany) with chloroform as solvent. Fouriertransform infrared (FTIR) spectra of all samples were measured at $400-4000 \mathrm{~cm}^{-1}$ using pressed $\mathrm{KBr}$ pellets on a spectrophotometer (Nicolet 6700, Thermo Nicolet, USA; Resolution: $2 \mathrm{~cm}^{-1}$ ). Uniaxial compression-decompression tests were carried out with a material tester (XBD-4000, Xinbiao Corp., China). The crosshead speed was $0.5 \mathrm{~mm} \mathrm{~min}^{-1}$ for compression and $0.2 \mathrm{~mm} \mathrm{~min}^{-1}$ for decompression. The surface structure and morphology of specimens were observed using a field-emission scanning electron microscope (FESEM; MRIA3, TESCAN, Czech) operated at $20 \mathrm{kV}$. The samples were sputter covered with a gold alloy prior to imaging. Nitrogen adsorption-desorption isotherms were calculated at $77 \mathrm{~K}$ through a Quantachrome ASiQwin surface area and porosimetry analyzer. Aerogel powders were degassed under vacuum at $80{ }^{\circ} \mathrm{C}$ for $8 \mathrm{~h}$ prior to the analysis. The SSA of aerogels was obtained from the adsorption isotherm using the BrunauerEmmett-Teller (BET) theory through an automatic surface area analyzer (Autosorb-iQ ${ }_{2}-\mathrm{MP}$, Quantachrome, USA) at $\mathrm{N}_{2}$ environment. Pore size distribution was obtained from desorption branches of the isotherms by applying the Barret-JoynerHalenda (BJH) method. For fully characterizing the pore structure, the pore size distribution was also measured by mercury intrusion porosimetry (MIP; AutoPore IV 9510, Micromeritics, USA). The thermal conductivity of aerogels was tested by using a Hot Disk thermal constants analyzer (TPS2500S, Hot Disk, Sweden) with the c5501 detector. And the thermal conductivity of PVPMSAs with the size of $\varnothing 30 \times 20 \mathrm{~mm}$ was measured under "bulk" mode.

\section{Results and discussion}

\subsection{Formation of monolithic PVPMSAs}

The preparation of monolithic PVPMSAs usually involves two processes, which are shown in Fig. 1. One is the creation of polymer PVMDMS with long-chain aliphatic hydrocarbons via free radical polymerization. The other is the formation of polyvinylpolymethylsiloxane gels by hydrolytic polycondensation. After these two processes, PVPMSAs were obtained by supercritical drying, the macroscopic photographs of which are shown in Fig. 1c. The infrared spectra (Fig. 2) show that the absorption peaks of vinyl functional groups ${ }^{22}(\mathrm{C}=\mathrm{C}$ stretching at $1600 \mathrm{~cm}^{-1},=\mathrm{C}-\mathrm{H}$ stretching at $3062 \mathrm{~cm}^{-1}$, out-of-plane swaying at $960 \mathrm{~cm}^{-1}$ and bending at $530 \mathrm{~cm}^{-1}$ ) disappeared after a long time of free radical polymerization, and PVMDMS with a high molecular weight was obtained. Next, polyorganosiloxane $^{25}$ ( $\mathrm{Si}-\mathrm{O}-\mathrm{Si}$ stretching at $1100-1000 \mathrm{~cm}^{-1}$ ) was formed by using the strong base of TMAOH as the catalyst through a sol-gel process. All these results obtained from Fig. 2 can be utilized to prove that we have prepared PVPMSAs. Furthermore, PVPMSAs exhibit excellent mechanical flexibility due to the double cross-linked structure. ${ }^{22}$ It can be observed from Fig. 3 that the mechanical properties of PVPMSAs are mainly related to density. PVPMSA with high density has relatively better elasticity and compression strength. For example, the Young's modulus of PVPMSA1 with a density of $0.277 \mathrm{~g} \mathrm{~cm}^{-3}$ has reached up to $11.36 \mathrm{MPa}$. As heat insulators, these aerogel materials can maintain monolithic shapes in actual operation, although they could not rebound smoothly to their original height after the force is removed at the ambient environment.

Compared with the brittle $\mathrm{SiO}_{2}$ aerogels, the reason why PVPMSAs do not crack could be attributed to two sides. The first and most important factor is that long-chain aliphatic hydrocarbons are beneficial to enhance the link strength between particles, thickening the skeleton structure of aerogels and preventing the crack of aerogels in drying. The other is using strong base TMAOH as the catalyst, which could make the sol rapidly to form the gel in a short time to prevent the growth of 


\section{a) Radical polymerization}
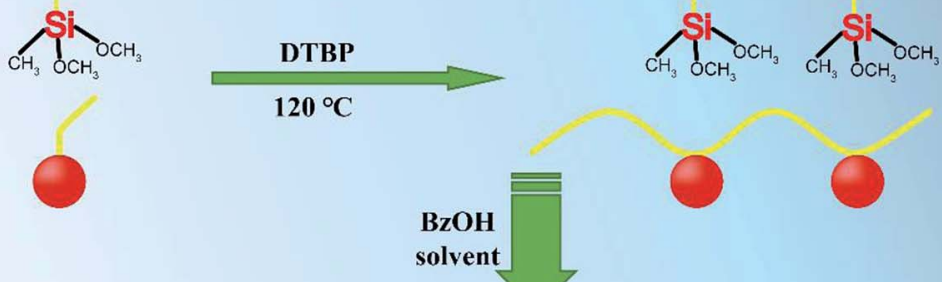

b) Hydrolytic polycondensation

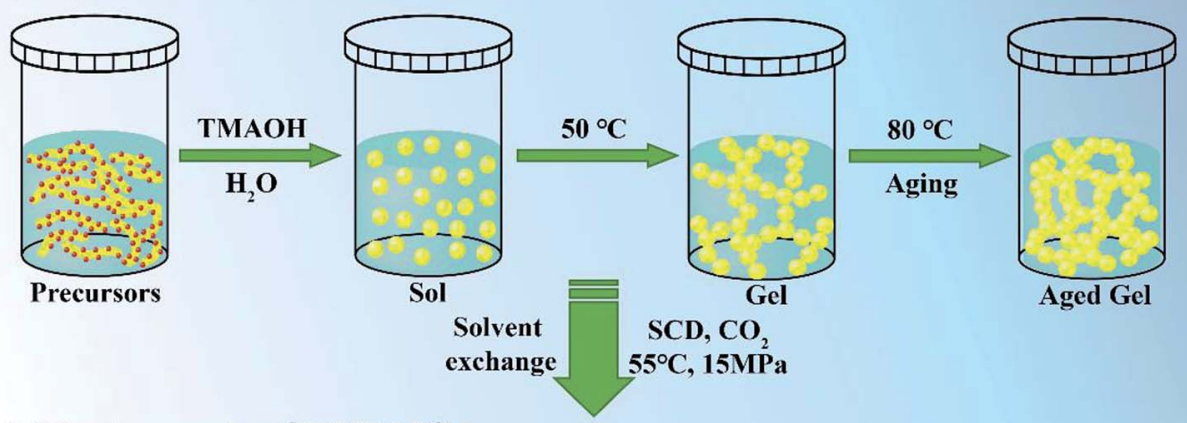

c) Photograph of PVPMSAs

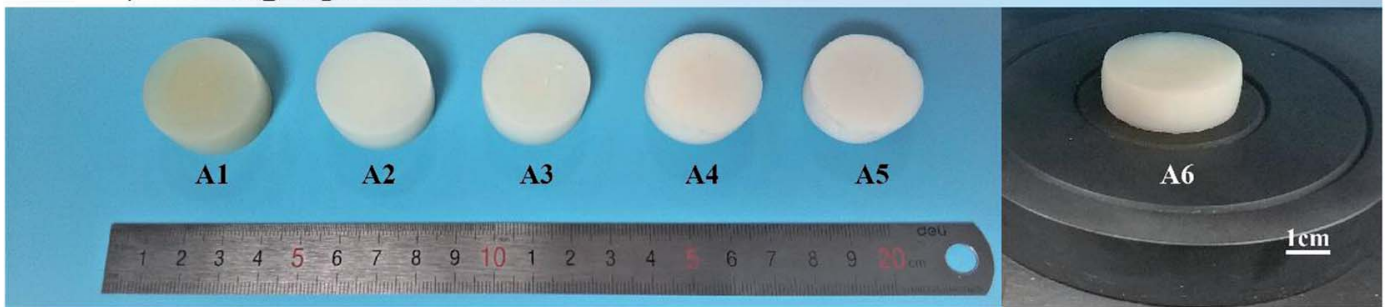

Fig. 1 Schematic diagram of synthetic process of PVPMSAs. (a) Radical polymerization; (b) hydrolytic polycondensation; (c) PVPMSAs obtained via $\mathrm{CO}_{2}$ supercritical drying.

secondary particles. As shown in Fig. 4, small particles possess a relatively large surface area, which could significantly expand the contact region of the pearl-like neck between particles and

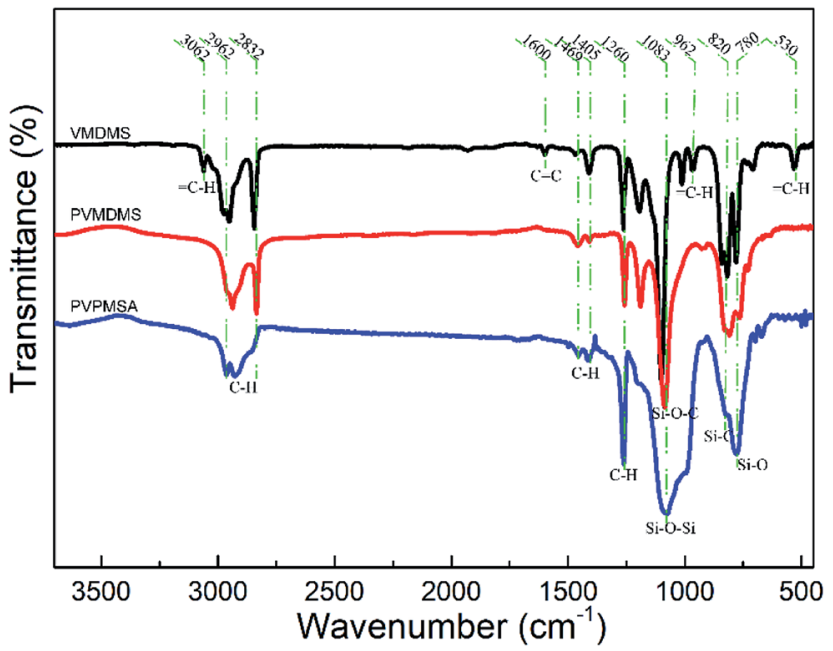

Fig. 2 FTIR spectra of VMDMS, PVMDMS and PVPMSA. favor to form a more stable skeleton structure after the gel aging. These two explanations are also fully confirmed by the SEM of PVPMSA6 from Fig. $4 \mathrm{~g}$ and $\mathrm{h}$. After compression, the

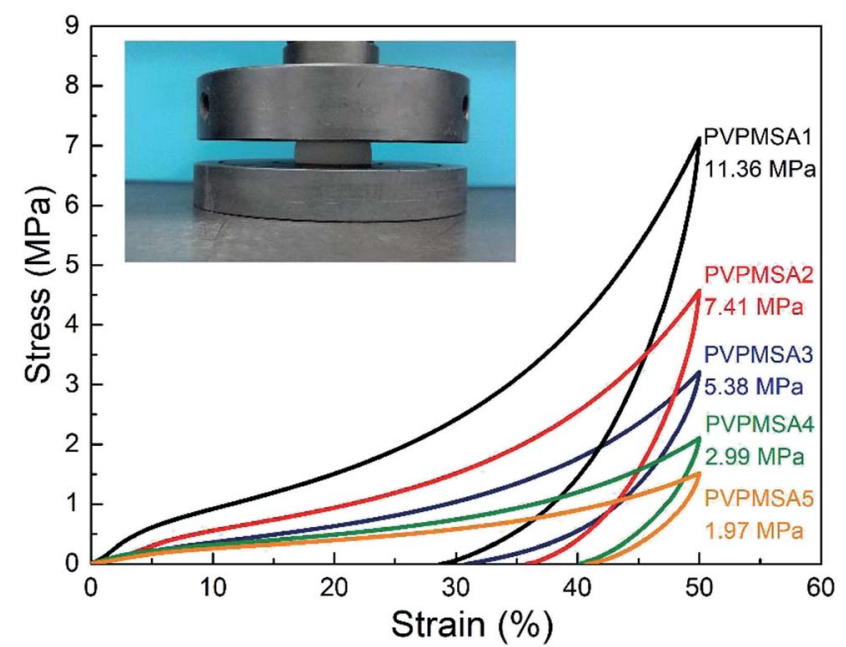

Fig. 3 Stress-strain curves of uniaxial compression-decompression tests on PVPMSAs. Values in this figure are the obtained Young's moduli. 

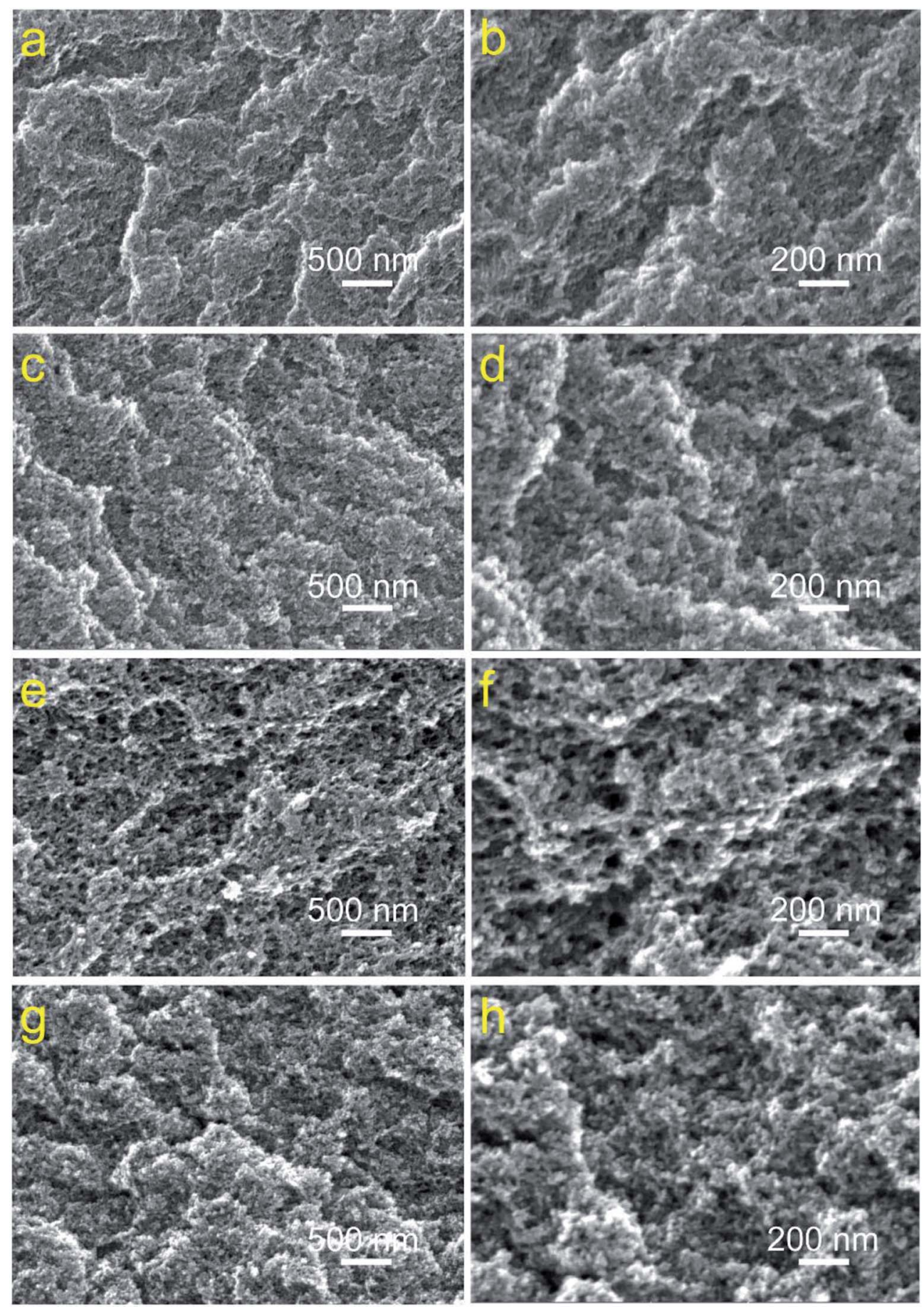

Fig. 4 SEM images of PVPMSAs synthesized by varying BzOH solvent contents. ( $a$ and b) PVPMSA1; (c and d) PVPMSA3; (e and f) PVPMSA5; ( $g$ and h) PVPMSA6.

microstructure of PVPMSA6 does not collapse, and the threedimensional porous morphology still appeared, which can be verified by the pore size distribution in Fig. $5 \mathrm{~d}$ and $\mathrm{f}$. There is no significant change in the pore sizes of PVPMSA3 and PVPMSA6 before and after compression. Fig. 4a-f show SEM images of PVPMSAs with density ranged from 0.177 to $0.277 \mathrm{~g} \mathrm{~cm}^{-3}$. Benefiting from the fine solubility of PVMDMS in $\mathrm{BzOH}$ solvent, the varied solvent addition only plays a role in regulating pore size and skeleton thickness, which has little effect on forming secondary particles. In fact, the growth of large particles has been inhibited due to strong base catalysis in the sol-gel process. In addition, the increased solvent dosage also results in a loosely porous skeleton texture, especially PVPMSA5 in Fig. 4e and f. The compressive strength and Young's modulus of PVPMSA5 are lower than those of other samples with high density. 

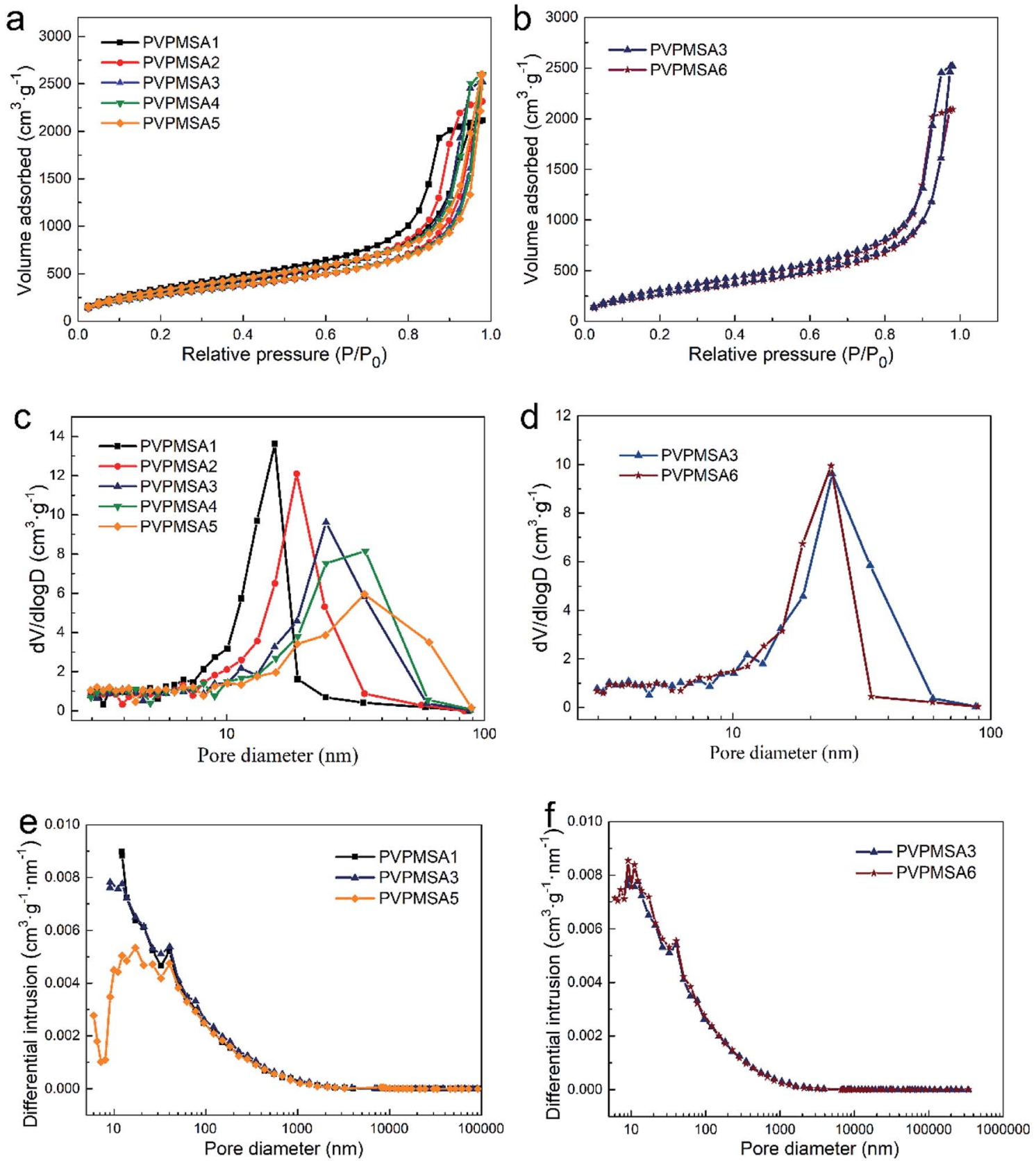

Fig. 5 Porous structure of PVPMSAs. (a and b) $\mathrm{N}_{2}$ adsorption-desorption isotherms; ( $c$ and d) pore size distributions of PVPMSAs obtained via $\mathrm{BJH}$ method; (e and f) pore size distributions of PVPMSAs measured by MIP.

\subsection{Porous structure features}

The typical sol-gel preparation process is the most crucial step in determining the high SSA of PVPMSAs. In this process, the hydrolytic polycondensation of PVPMSAs could be facilely achieved by the catalysis of strong base TMAOH, then the sol to gel phase can be transformed rapidly in a short time. Herein, due to the quick gel effect, the growth of small particles in the sol phase is effectively suppressed. As we know, smaller particles would bring a larger SSA, which is why PVPMSAs possess a high SSA. As shown in Table 1, the SSA of PVPMSA1 measured by the BET method is up to $1218 \mathrm{~m}^{2} \mathrm{~g}^{-1}$, which is generally higher than the SSA of other polymer-based aerogels. ${ }^{26-29}$ Evidence from Table 1 also suggests that the SSA of PVPMSA2-5 does not vary regularly with the increased solvent dosage, but remains relatively stable at about $1050 \mathrm{~m}^{2} \mathrm{~g}^{-1}$. This result also proves that these small particles formed in the rapid gelation stage are crucially important for obtaining a huge SSA.

Fig. 5a and $\mathrm{b}$ exhibit the nitrogen adsorption-desorption isotherms of PVPMSAs. Within the relative pressure range of 0 0.7 , the nitrogen adsorption increases steadily and slowly, indicating that there is a large number of pores in PVPMSAs. Fig. $5 \mathrm{c}$ and $\mathrm{d}$ show the pore size distribution of PVPMSAs obtained by the $\mathrm{BJH}$ method. An interesting phenomenon is that there are many uniform nanopores in aerogel networks. The peaks of pore 
Table 1 Physical properties of doubly cross-linked PVPMSAs

\begin{tabular}{llllll}
\hline Sample & $\mathrm{BzOH} / \mathrm{Si}\left(\mathrm{mol} \mathrm{mol}^{-1}\right)$ & $\rho\left(\mathrm{g} \mathrm{cm}^{-3}\right)$ & $\mathrm{SSA}^{a}\left(\mathrm{~m}^{2} \mathrm{~g}^{-1}\right)$ & $d^{b}\left(\mathrm{~nm}^{2}\right)$ & Pore volume $^{c}\left(\mathrm{~cm}^{3} \mathrm{~g}^{-1}\right)$ \\
\hline PVPMSA1 & 3.0 & 0.277 & 1218 & 15.35 & 3.278 \\
PVPMSA2 & 4.0 & 0.238 & 1083 & 18.66 & 3.585 \\
PVPMSA3 & 5.0 & 0.221 & 1079 & 24.25 & 3.900 \\
PVPMSA4 & 6.0 & 0.198 & 1079 & 34.49 & 4.010 \\
PVPMSA5 & 7.0 & 0.177 & 1093 & 34.19 & 4.020 \\
PVPMSA6 & 5.0 & 0.319 & 1055 & 24.03 & 3.241
\end{tabular}

${ }^{a}$ SSA obtained from nitrogen adsorption isotherm. ${ }^{b}$ Mean pore diameter obtained via BJH method. ${ }^{c}$ Pore volume acquired from $\mathrm{N}_{2}$ adsorptiondesorption isotherms.

size distribution are concentrated in the range of $15-35 \mathrm{~nm}$, which means that PVPMSAs with mesoporous structures would be fine materials for thermal insulation. However, these results of pore size distribution obtained from the BJH method are not well corresponding with that observed in Fig. 4a-f. There are still some macropores (50-1000 nm) measured by the MIP method in Fig. 5e and f. Obviously, the internal networks of PVPMSAs are composed of typically hierarchical pores, and mesopores occupy a large proportion. Furthermore, relying on the excellent solubility of PVMDMS in $\mathrm{BzOH}$ solvent, the mean pore size of PVPMSAs (Table 1) can be controlled by adjusting the solvent content. Meanwhile, the pore volume of PVPMSAs is also affected by pore size. Evidence from Table 1 shows that the pore volume increases from 3.278 to $4.020 \mathrm{~cm}^{3} \mathrm{~g}^{-1}$, which is mainly related with the pore size going to a higher degree.

\subsection{Thermal conductivity of PVPMSAs}

The total thermal conductivity $(\lambda)$ of PVPMSAs generally consists of three parts, including the solid thermal conductivity $\left(\lambda_{\mathrm{s}}\right)$, the gaseous thermal conductivity $\left(\lambda_{\mathrm{g}}\right)$, and the radiative thermal conductivity $\left(\lambda_{\mathrm{r}}\right)^{30-35}$

$$
\lambda=\lambda_{\mathrm{s}}+\lambda_{\mathrm{g}}+\lambda_{\mathrm{r}}
$$

For the solid thermal conductivity of PVPMSAs, it is mainly related to the density of aerogels. ${ }^{32}$

$$
\lambda_{\mathrm{s}}=C \rho^{\alpha}
$$

where the constant $C$ is depended on the interconnectivity of the particles in aerogel, $\alpha \approx 1.5$ was obtained for both $\mathrm{SiO}_{2}$ and carbon aerogels, and $\rho$ is the density of aerogel. The radiation thermal conductivity of PVPMSAs is mainly related to temperature and density. ${ }^{33,34}$

$$
\lambda_{\mathrm{r}}=\frac{16 \sigma n^{2} T^{3}}{3 \rho e(T)}
$$

Here $\sigma$ is the Stefan-Boltzman constant, $n$ is the mean index of refraction of the specimen, $T$ is the absolute temperature, $e(T)$ is the mass specific extinction coefficient, and $\rho$ is the density of aerogel.

Finally, the gaseous thermal conductivity can be expressed as follows: $:^{32,34}$

$$
\lambda_{\mathrm{g}}=\Phi \frac{\lambda_{\mathrm{g}, 0}}{1+2 \beta l P_{0} /(P D)}
$$

where $\Phi$ is the porosity, $P_{0}$ the reference gas pressure, $P$ the gas pressure, $\lambda_{g, 0}$ the thermal conductivity of the non-convectional free gas at $P_{0}, \beta$ is a constant including the interaction between the gas molecules and the pore walls, $l$ is the mean free path of gas molecules at $P_{0}$, and $D$ the average pore size of the porous material.

When the density of PVPMSAs is controlled to $0.177 \mathrm{~g} \mathrm{~cm}^{-3}$, $\lambda$ is $21.02 \mathrm{~mW} \mathrm{~m}^{-1} \mathrm{~K}^{-1}$ at $25{ }^{\circ} \mathrm{C}$ and ambient pressure. And Fig. 6 reveals that there is a slow rise in $\lambda$ of PVPMSAs with the density varied from 0.177 to $0.277 \mathrm{~g} \mathrm{~cm}^{-3}$. Compared with $\lambda$ at ambient pressure, $\lambda$ at $5 \mathrm{~Pa}$ also shows an increasing trend. What's more, $\lambda_{\mathrm{g}}$ is calculated by subtracting $\lambda$ of 5 Pa from $\lambda$ at ambient pressure, which is obviously lower than the air thermal conductivity of $26 \mathrm{~mW} \mathrm{~m}{ }^{-1} \mathrm{~K}^{-1}$, 34 showing extremely low gaseous thermal conductivity of PVPMSAs. Thus, these results obtained above suggest that $\lambda$ of PVPMSAs mainly comes from $\lambda_{\mathrm{s}}$ and $\lambda_{\mathrm{r}}$, and $\lambda_{\mathrm{g}}$ accounts for a small part.

For the sample PVPMSA3, the density increases from 0.221 to $0.319 \mathrm{~g} \mathrm{~cm}^{-3}$ after compression test. On the contrary, the pore volume of PVPMSA3 decreases from 3.900 to $3.241 \mathrm{~cm}^{3} \mathrm{~g}^{-1}$ in Table 1, which makes $\lambda$ increase from 21.97 to $27.19 \mathrm{~mW} \mathrm{~m}^{-1}$ $\mathrm{K}^{-1}$ and $\lambda_{\mathrm{s}}+\lambda_{\mathrm{r}}$ increase slowly from 16.20 to $23.44 \mathrm{~mW} \mathrm{~m}^{-1} \mathrm{~K}^{-1}$. Eqn (2) validates the growth trend of thermal conductivity, and the results of the correlational analysis are shown in Fig. 7 . Meanwhile, according to eqn (3), the change in density also

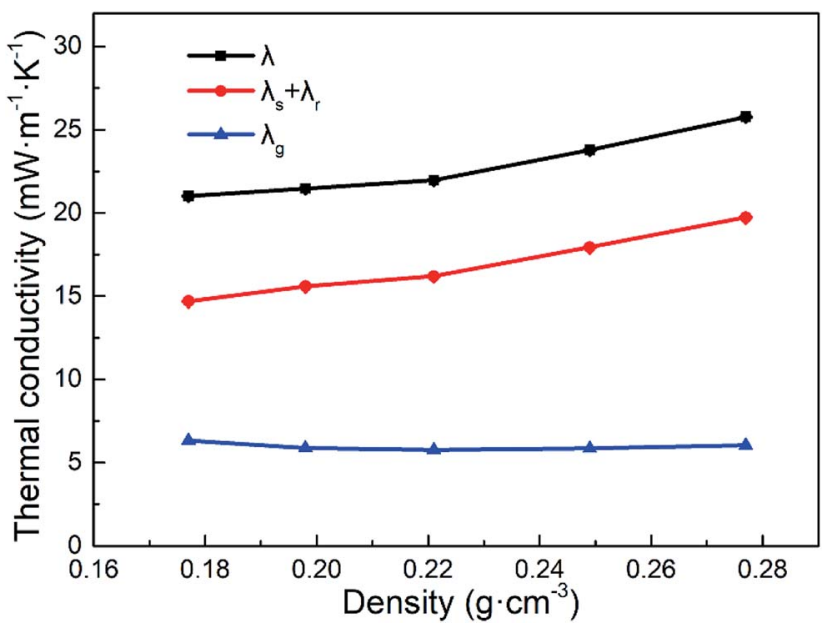

Fig. 6 Thermal conductivity of PVPMSAs under different conditions. $\lambda$ was obtained at $25^{\circ} \mathrm{C}$ under ambient pressure. $\lambda_{s}+\lambda_{r}$ was obtained at $25^{\circ} \mathrm{C}$ in $5 \mathrm{~Pa}$. $\lambda_{\mathrm{g}}$ was acquired by using eqn (1). 


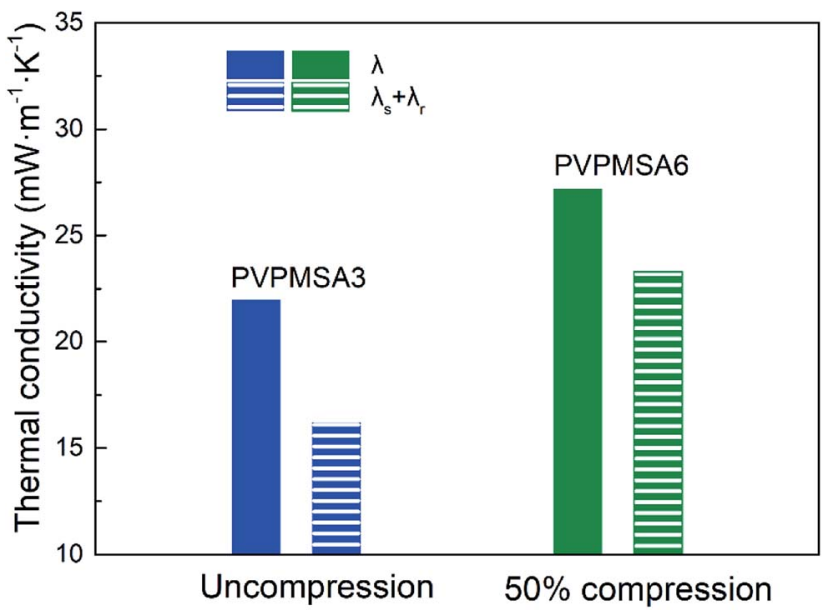

Fig. 7 Thermal conductivity of PVPMSAs under different compression states. PVPMSA6 was obtained from PVPMSA3 after $50 \%$ uniaxial strain compression.

leads to a decrease in $\lambda_{\mathrm{r}}$ at a given temperature, but $\lambda$ shows the opposite growth rule, which means that the density has a great effect on $\lambda_{\text {s }}$. Fig. 8 shows that there is a steady growth in $\lambda$ with temperature ranged from 25 to $90^{\circ} \mathrm{C}$, and we can use eqn (3) to explain this appearance. For sample PVPMSA3, at a given density, $\lambda$ increases from $21.97 \mathrm{~mW} \mathrm{~m}^{-1} \mathrm{~K}^{-1}$ at $25{ }^{\circ} \mathrm{C}$ to 27.04 $\mathrm{mW} \mathrm{m}{ }^{-1} \mathrm{~K}^{-1}$ at $90{ }^{\circ} \mathrm{C}$, which is mainly contributed by $\lambda_{\mathrm{r}}$. In general, these results in the Fig. 8 indicate that there is a positive correlation between $\lambda_{\mathrm{r}}$ and temperature, and PVPMSAs still have excellent thermal insulation properties at $90{ }^{\circ} \mathrm{C}$.

With the mean pore size of PVPMSAs increasing from 15 to $35 \mathrm{~nm}, \lambda_{\mathrm{g}}$ is maintained at about $5 \mathrm{~mW} \mathrm{~m}{ }^{-1} \mathrm{~K}^{-1}$ to $6 \mathrm{~mW} \mathrm{~m}^{-1}$ $\mathrm{K}^{-1}$ in the Fig. 6 , which means that smaller pore size is more conducive to improving thermal insulation performance. Fig. 9 shows the relationship between $\lambda$ and gas pressure, and $\lambda$ of PVPMSA3 is $21.97 \mathrm{~mW} \mathrm{~m}^{-1} \mathrm{~K}^{-1}$ at $25{ }^{\circ} \mathrm{C}$ and ambient pressure. With the air pressure decreasing, $\lambda$ decreases continuously, which is also verified by eqn (4). Thus, it can be concluded that gas pressure is an important factor to affect $\lambda_{\mathrm{g}}$ at a given temperature. Fig. 10 presents $\lambda$ under different atmospheric

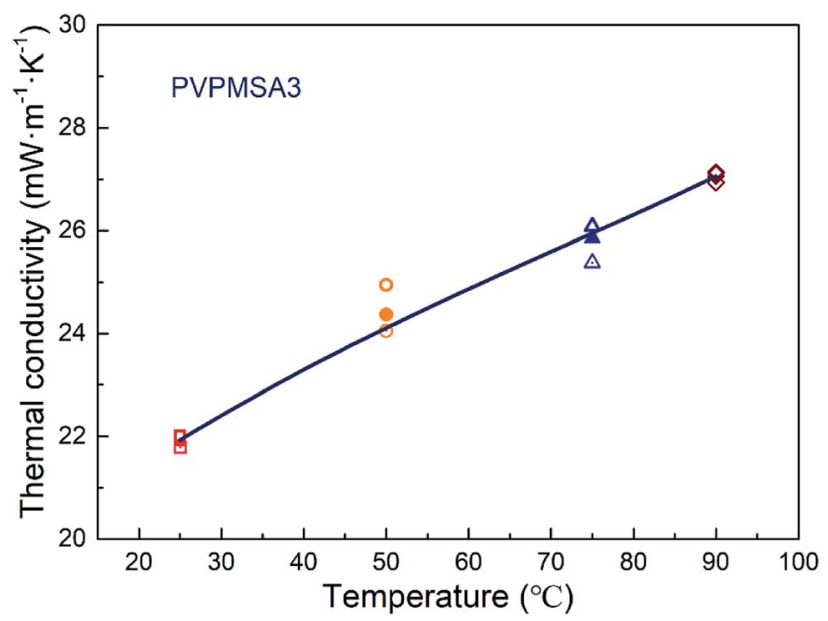

Fig. 8 Thermal conductivity of PVPMSA3 under different temperature.

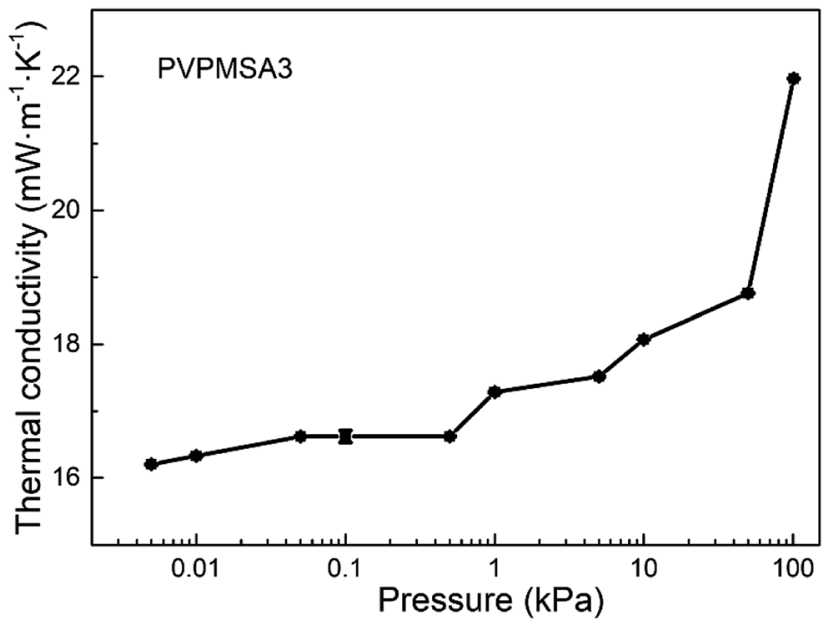

Fig. 9 Thermal conductivity of PVPMSA3 in varying gas pressure.

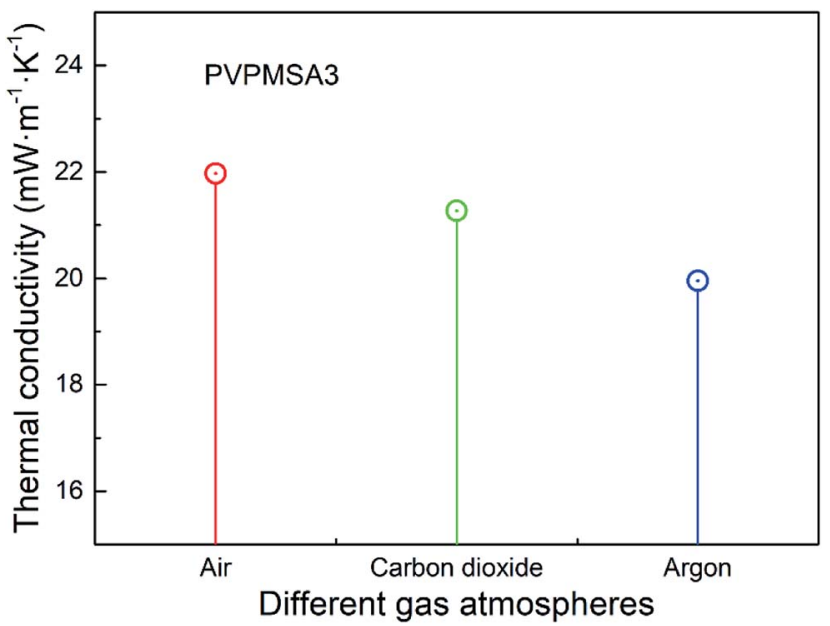

Fig. 10 Thermal conductivity of PVPMSA3 at different gas atmospheres.

conditions. It was found that $\lambda$ of PVPMSA3 was $21.27 \mathrm{~mW} \mathrm{~m}^{-1}$ $\mathrm{K}^{-1}$ in the carbon dioxide atmosphere and $19.96 \mathrm{~mW} \mathrm{~m}^{-1} \mathrm{~K}^{-1}$ in the argon atmosphere, both of which were lower than those in an air atmosphere. A possible explanation for this phenomenon might be that $\lambda_{\mathrm{g}}$ is closely associated with the composition of the gas and the free path of the gas molecules. Generally, gas with a simple composition or a long molecular free path exhibits a relatively low gaseous heat transfer capability. ${ }^{36}$

\section{Conclusions}

The organic-inorganic hybrid PVPMSAs with a double crosslinking structure were prepared by free radical polymerization and hydrolytic polycondensation. And the doubly crosslinked structure composed of small nanoscale particles gives PVPMSAs a high SSA, reaching up to $1218 \mathrm{~m}^{2} \mathrm{~g}^{-1}$. By adjusting solvent dosage, the pore size is concentrated in the range of $15-35 \mathrm{~nm}$, which effectively limits the heat transfer of gas in PVPMSAs, and makes PVPMSAs exhibit high gas insulation performance. On 
this basis, the thermal conductivity of PVPMSAs was studied systematically. It was found that the lowest thermal conductivity $\left(\lambda=14.69 \mathrm{~mW} \mathrm{~m}{ }^{-1} \mathrm{~K}^{-1}\right.$ at $25{ }^{\circ} \mathrm{C}$ in $\left.5 \mathrm{~Pa}\right)$ was obtained for PVPMSAs with a density of $0.177 \mathrm{~g} \mathrm{~cm}^{-3}$, and the thermal conductivity could be changeable regularly with the variation of conditions, such as density, gas pressure, and temperature. Meanwhile, the external atmosphere would have a significant impact on the thermal conductivity of aerogels. The thermal conductivity of carbon dioxide and argon atmosphere is lower than that at ambient conditions. In conclusion, all these results would help us to have a better understanding of the heat transfer process in PVPMSAs, so as to design more highperformance insulation materials, which would be ideal insulators for space, architecture, and house in the future.

\section{Conflicts of interest}

There are no conflicts to declare.

\section{Acknowledgements}

This work was supported by National Natural Science Foundation of China (Grant no. 51302317, 51712360) and Natural Science Foundation of Hunan Province (Grant no. 14JJ3008). The authors gratefully acknowledged the generous support from the Program for Science and Technology Innovative Team in Colleges of Hunan Province, and the Program for Technology Innovative Group of National University of Defense Technology.

\section{References}

1 T. Gao, B. P. Jelle, T. Ihara, et al. Insulating glazing units with silica aerogel granules: the impact of particle size, Appl. Energy, 2014, 128, 27-34.

2 A. C. Pierre and G. M. Pajonk, Chemistry of aerogels and their applications, Chem. Rev., 2002, 102(11), 4243-4266.

3 H. Rotter, M. V. Landau, M. Carrera, et al. High surface area chromia aerogel efficient catalyst and catalyst support for ethylacetate combustion, Appl. Catal., B, 2004, 47(2), 111126.

4 L. Piao, Y. Li, J. Chen, et al. Methane decomposition to carbon nanotubes and hydrogen on an alumina supported nickel aerogel catalyst, Catal. Today, 2002, 74(1-2), 145-155.

5 J. T. Korhonen, M. Kettunen, R. H. A. Ras, et al. Hydrophobic nanocellulose aerogels as floating, sustainable, reusable, and recyclable oil absorbents, ACS Appl. Mater. Interfaces, 2011, 3(6), 1813-1816.

6 J. Li, J. Li, H. Meng, et al. Ultra-light, compressible and fireresistant graphene aerogel as a highly efficient and recyclable absorbent for organic liquids, J. Mater. Chem. A, 2014, 2(9), 2934-2941.

7 J. L. Gurav, A. V. Rao, D. Y. Nadargi, et al. Ambient pressure dried TEOS-based silica aerogels: good absorbents of organic liquids, J. Mater. Sci., 2010, 45(2), 503.

8 C. Buratti and E. Moretti, Glazing systems with silica aerogel for energy savings in buildings, Appl. Energy, 2012, 98, 396403.
9 M. Mirzaeian and P. J. Hall, Preparation of controlled porosity carbon aerogels for energy storage in rechargeable lithium oxygen batteries, Electrochim. Acta, 2009, 54(28), 7444-7451.

10 B. Fang and L. Binder, A modified activated carbon aerogel for high-energy storage in electric double layer capacitors, J. Power Sources, 2006, 163(1), 616-622.

11 C. A. García-González, M. Alnaief and I. Smirnova, Polysaccharide-based aerogels-Promising biodegradable carriers for drug delivery systems, Carbohydr. Polym., 2011, 86(4), 1425-1438.

12 I. Smirnova, S. Suttiruengwong and W. Arlt, Feasibility study of hydrophilic and hydrophobic silica aerogels as drug delivery systems, J. Non-Cryst. Solids, 2004, 350, 54-60.

13 Z. Ulker and C. Erkey, An emerging platform for drug delivery: aerogel based systems, J. Controlled Release, 2014, 177, 51-63.

14 T. M. Tillotson, J. F. Poco and L. W. Hrubesh, et al. Method for producing metal oxide aerogels having densities less than $0.02 \mathrm{~g} \mathrm{~cm}^{-3}$, US Pat., 5275796, 1994-1-4.

15 M. W. Droege, P. R. Coronado and L. M. Hair. Method for making monolithic metal oxide aerogels, US Pat., 5395805, 1995-3-7.

16 K. Kanamori, R. Ueoka, T. Kakegawa, et al. Hybrid silicone aerogels toward unusual flexibility, functionality, and extended applications, J. Sol-Gel Sci. Technol., 2018, 1-10.

17 S. Zhao, Z. Zhang, G. Sèbe, et al. Multiscale assembly of superinsulating silica aerogels within silylated nanocellulosic scaffolds: improved mechanical properties promoted by nanoscale chemical compatibilization, Adv. Funct. Mater., 2015, 25(15), 2326-2334.

18 D. Shi, Y. Sun, J. Feng, et al. Experimental investigation on high temperature anisotropic compression properties of ceramic-fiber-reinforced $\mathrm{SiO}_{2}$ aerogel, J. Mater. Sci. Eng. A, 2013, 585, 25-31.

$19 \mathrm{H}$. Wu, Y. Chen, Q. Chen, et al. Synthesis of flexible aerogel composites reinforced with electrospun nanofibers and microparticles for thermal insulation, J. Nanomater., 2013, 2013, 10.

20 K. Kanamori and K. Nakanishi, Controlled pore formation in organotrialkoxysilane-derived hybrids: from aerogels to hierarchically porous monoliths, Chem. Soc. Rev., 2011, 40(2), 754-770.

21 T. Shimizu, K. Kanamori and K. Nakanishi, Silicone-based organic-inorganic hybrid aerogels and xerogels, Chem.Eur. J., 2017, 23(22), 5176-5187.

22 G. Zu, T. Shimizu, K. Kanamori, et al. Transparent, superflexible doubly cross-linked polyvinylpolymethylsiloxane aerogel superinsulators via ambient pressure drying, ACS Nano, 2018, 12(1), 521-532.

23 G. Zu, K. Kanamori, T. Shimizu, et al. Versatile double-crosslinking approach to transparent, machinable, supercompressible, highly bendable aerogel thermal superinsulators, Chem. Mater., 2018, 30(8), 2759-2770.

24 G. Zu, K. Kanamori, A. Maeno, et al. Superflexible multifunctional polyvinylpolydimethylsiloxane-based aerogels as efficient absorbents, thermal superinsulators, 
and strain Sensors, Angew. Chem., Int. Ed., 2018, 57(31), 9722-9727.

25 H. S. Yang, S. Y. Choi, S. H. Hyun, et al. Ambient-dried low dielectric $\mathrm{SiO}_{2}$ aerogel thin film, J. Non-Cryst. Solids, 1997, 221(2-3), 151-156.

26 H. Sehaqui, Q. Zhou and L. A. Berglund, High-porosity aerogels of high specific surface area prepared from nanofibrillated cellulose (NFC), Compos. Sci. Technol., 2011, 71(13), 1593-1599.

27 H. Sehaqui, Q. Zhou, O. Ikkala, et al. Strong and tough cellulose nanopaper with high specific surface area and porosity, Biomacromolecules, 2011, 12(10), 3638-3644.

28 H. Guo, M. A. B. Meador, L. McCorkle, et al. Polyimide aerogels cross-linked through amine functionalized polyoligomeric silsesquioxane, ACS Appl. Mater. Interfaces, 2011, 3(2), 546-552.

29 S. Zhang, J. Feng, J. Feng, et al. Formation of enhanced gelatum using ethanol/water binary medium for fabricating chitosan aerogels with high specific surface area, Chem. Eng. J., 2017, 309, 700-707.

30 X. Lu, O. Nilsson, J. Fricke, et al. Thermal and electrical conductivity of monolithic carbon aerogels, J. Appl. Phys., 1993, 73(2), 581-584.
31 X. Lu, P. Wang, M. C. Arduini-Schuster, et al. Thermal transport in organic and opacified silica monolithic aerogels, J. Non-Cryst. Solids, 1992, 145(145), 207-210.

32 X. Lu, M. C. Arduini-Schuster, J. Kuhn, et al. Thermal conductivity of monolithic organic aerogels, Science, 1992, 255(5047), 971-972.

33 G. Hayase, K. Kugimiya, M. Ogawa, et al. The thermal conductivity of polymethylsilsesquioxane aerogels and xerogels with varied pore sizes for practical application as thermal superinsulators, J. Mater. Chem. A, 2014, 2(18), 6525-6531.

34 X. Lu, R. Caps, J. Fricke, et al. Correlation between structure and thermal conductivity of organic aerogels, J. Non-Cryst. Solids, 1995, 188(3), 226-234.

35 X. Lu, P. Wang, M. C. Arduini-Schuster, et al. Thermal transport in organic and opacified silica monolithic aerogels, J. Non-Cryst. Solids, 1992, 145, 207-210.

36 J. Feng, X. Wang, Y. Jiang, et al. Study on thermal conductivities of aromatic polyimide aerogels, ACS Appl. Mater. Interfaces, 2016, 8(20), 12992-12996. 